\title{
Early Start of Chemotherapy after Resection of Brain Metastasis from Colon Cancer with Synchronous Multiple Liver Metastases
}

\author{
Yoichiro Yoshida Seiichiro Hoshino Toru Miyake \\ Sho Fukuda Kazunosuke Yamada Aisu Naoya \\ Syu Tanimura Yuichi Yamashita \\ Department of Gastroenterological Surgery, Fukuoka University \\ Faculty of Medicine, Fukuoka, Japan
}

Key Words

Colorectal cancer $\cdot$ Chemotherapy $\cdot$ Brain metastasis · Surgery $\cdot$ XELOX

\begin{abstract}
Brain metastasis (BM) is infrequent in colorectal cancer (CRC) patients. Although BM from CRC is a late-stage phenomenon with an extremely poor prognosis, some subsets of patients would benefit from a multidisciplinary management strategy. The prognosis of patients with BM from CRC is associated with the curability of the therapy for BM and number of metastatic organs. The start of chemotherapy treatment usually requires a delay of about 4 weeks after surgical resection in patients with primary CRC having synchronous distant metastasis. However, there is no evidence to indicate the required length of this delay interval. In addition, there is a chance that a patient may die because postoperative chemotherapy was not started soon enough and a metastatic tumor was able to develop rapidly. Here, we present a case where combination chemotherapy with capecitabine and oxaliplatin (XELOX) was started within 1 week after resection of BM from colon cancer for synchronous multiple liver metastases. To our knowledge, this is the first report of the start of chemotherapy, involving treatments such as folinic acid, fluorouracil, and oxaliplatin (FOLFOX); folinic acid, fluorouracil, and irinotecan (FOLFIRI); and XELOX within 1 week after resection of BM from colon cancer with synchronous multiple liver metastases. These findings suggest possible changes in the start time of chemotherapy after surgery in the future.
\end{abstract}




\section{Introduction}

Brain metastasis (BM) is infrequent in colorectal cancer (CRC) patients. BM was diagnosed in $8.5 \%$ of patients in a recent cohort study; the cumulative incidence of BM after 5 years was estimated at $16.3 \%$ in patients with lung cancer, $9.8 \%$ in patients with renal cell carcinoma, $7.4 \%$ in patients with melanoma, $5.0 \%$ in patients with breast cancer and $1.2 \%$ in patients with CRC [1]. Although BM from CRC is a late-stage phenomenon with an extremely poor prognosis, some subsets of patients would benefit from a multidisciplinary management strategy. The prognosis of patients with BM from CRC is associated with the curability of the therapy for BM and number of metastatic organs [2].

Surgical resection of asymptomatic primary CRC with unresectable synchronous metastases is controversial. There is no doubt that, among patients with severe intestinal symptoms, resection is mandatory before starting systemic chemotherapy [3-5]. Palliative resection of the primary tumor is also reported to improve the efficacy of systemic chemotherapy [6] and prolong the duration of chemotherapy [7]. A recent review article suggested that non-curative resection of asymptomatic colorectal primary tumors may prolong survival in patients with metastatic CRC [8]. On the other hand, another article concluded that initial chemotherapy should be started, with resection of the primary tumor reserved for the small proportion of patients who develop major complications from the primary tumor, because resection of an asymptomatic primary tumor provides only minimal palliative benefit [9].

Surgical resection may delay the start of chemotherapy [10]. Generally, an interval of 4 weeks is considered necessary after an operation until the beginning of chemotherapy treatments such as folinic acid, fluorouracil, and oxaliplatin (FOLFOX); folinic acid, fluorouracil, and irinotecan (FOLFIRI); and capecitabine and oxaliplatin (XELOX); however, there is no apparent evidence for this delay. A metastatic tumor can enlarge rapidly before the start of chemotherapy and possibly lead to patient death. Because the significance of the postoperative 4-week delay until the start of chemotherapy is not clear, we performed an early chemotherapy start in a patient who had undergone resection of BM from colon cancer for synchronous multiple liver metastases.

\section{Case Report}

A 62-year-old man was referred to our hospital with abdominal fullness and gait disturbance. Barium enema revealed a nearly obstructing tumor in the rectum (fig. 1). Stenosis prevented the endoscope from passing through. The patient needed a fasting because of the stenosis of the rectum. Abdominal computed tomography (CT) revealed more than 10 hepatic metastases (fig. $2 \mathrm{a}$ ). Head CT and MRI showed a cerebellum metastasis ( $\underline{\text { fig. } 3}$ ). The patient underwent resection of BM and loop transverse colostomy. After surgery, the patient was allowed to drink water. On postoperative day 1 , the patient was offered a meal. His postoperative recovery was uneventful. The patient strongly hoped for an early start of chemotherapy on postoperative day 5. Therefore, we started XELOX therapy on postoperative day 7 [oxaliplatin $\left(130 \mathrm{mg} / \mathrm{m}^{2}\right)$ on day 1 plus capecitabine $\left(1,000 \mathrm{mg} / \mathrm{m}^{2}\right)$ twice daily on days 1-14, every 3 weeks]. The patient was subsequently discharged in good condition on postoperative day 16 . Gait disturbance was completely recovered 2 weeks after the treatment. XELOX + bevacizumab [BV $(7.5 \mathrm{mg} / \mathrm{kg})$ and oxaliplatin $\left(130 \mathrm{mg} / \mathrm{m}^{2}\right)$ on day 1 plus capecitabine $\left(1,000 \mathrm{mg} / \mathrm{m}^{2}\right)$ twice daily on days $1-14$, every 3 weeks] was administered on postoperative day 28 [11]. After 4 cycles of XELOX + BV, CT revealed a reduction in tumor size (fig. 2b). Laboratory studies 
revealed a serum carcinoembryonic antigen level of 3,460 ng/ml, which improved to $936 \mathrm{ng} / \mathrm{ml}$ after 4 cycles of chemotherapy. Observed adverse events that had appeared before were only a peripheral neuropathy in the acute stage (grade 1) and hand-foot syndrome (grade 1). Hematological toxicity, surgical site infection and incisional hernia were not noted.

Tumor progression has not occurred for 4 months; the patient is now undergoing XELOX + BV therapy.

\section{Discussion}

Patients with BM from CRC have a poor prognosis because they often have substantial extracranial metastatic disease. Traditionally, the therapeutic goal in many of these patients has been to palliate debilitating neurologic symptoms, because most of these patients die of systemic disease. However, new advances in metastatic CRC management - including the incorporation of monoclonal antibody therapies BV [12], cetuximab [13], and panitumumab [14] - are enhancing the outcomes of patients with systemic disease. Although these targeted therapies have improved the management of systemic disease, they are not as effective for BM given the restrictions on delivery into a tumor caused by the blood-tumor and blood-brain barriers. In light of these advances, the management of BM from CRC may deserve reconsideration.

It is fairly clear that careful patient selection influences the relative success of brain lesion resection. In a retrospective review from the late 1990s, Wronski and Arbit [15] evaluated the disease-specific outcomes of surgical resection in 73 patients with metastatic CRC to the brain who met specific criteria for surgery. The patients were evaluated for the extent of disease. Those with limited extracranial disease and a life expectancy in excess of 6 months were considered, as were those with a maximum of 2 to 3 accessible lesions.

Currently, the precise timing for starting treatment with chemotherapeutic agents prior to and/or after surgery in order to avoid postoperative complications is not clear, but an interval of at least 4 weeks has been suggested. In most clinical trials, patients who had undergone an operation within 4 weeks were excluded. In Benoist et al.'s study [10], the mean interval between diagnosis and chemotherapy start was 44 days in the resection group versus 15 days in the chemotherapy group. In this case, although the operation was performed, the interval between diagnosis and chemotherapy start was 14 days. Resection of the primary tumor significantly increases hospital stay and delays the initiation of chemotherapy; however, there is no evidence to suggest that this delay is associated with reduced response rates leading to curative resection or reduced survival. However, there is a chance that patients may die if they are not able to start chemotherapy because of the rapid postoperative progression of a metastatic tumor $[16,17]$. We have reported a case of an early chemotherapy start in a patient who had undergone a right hemicolectomy for synchronous multiple liver metastases. And he survived for 22 months despite the huge liver metastases [18]. An early start of chemotherapy after surgery is desirable not only for primary CRC but also for the metastatic site.

Resection of colorectal tumors with severe stenosis and bleeding is the first step of treatment in order to prevent the complications related to colorectal tumors. An early start of chemotherapy after surgery may be safe and improve the prognosis of colon 
cancer patients with synchronous metastases. Prospective studies are needed to confirm the effect and safety of an early start of chemotherapy after surgery. We have already started a phase II trial to examine patients receiving an early start of chemotherapy.

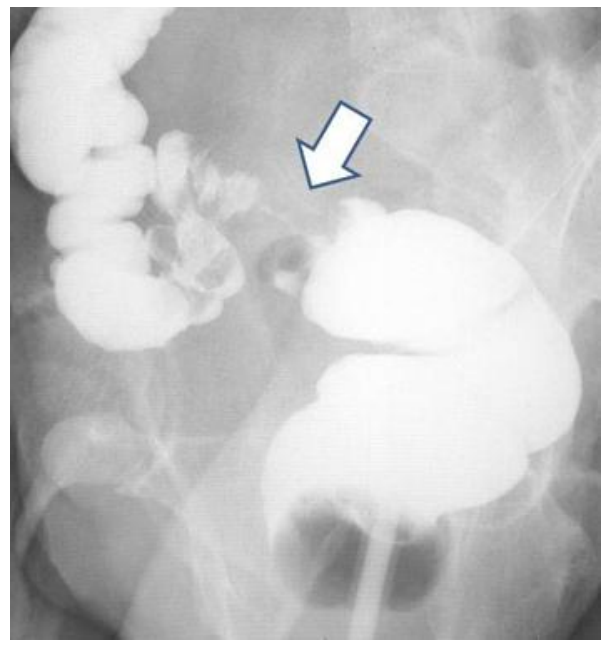

Fig. 1. Barium enema. The arrow indicates the nearly obstructing tumor.
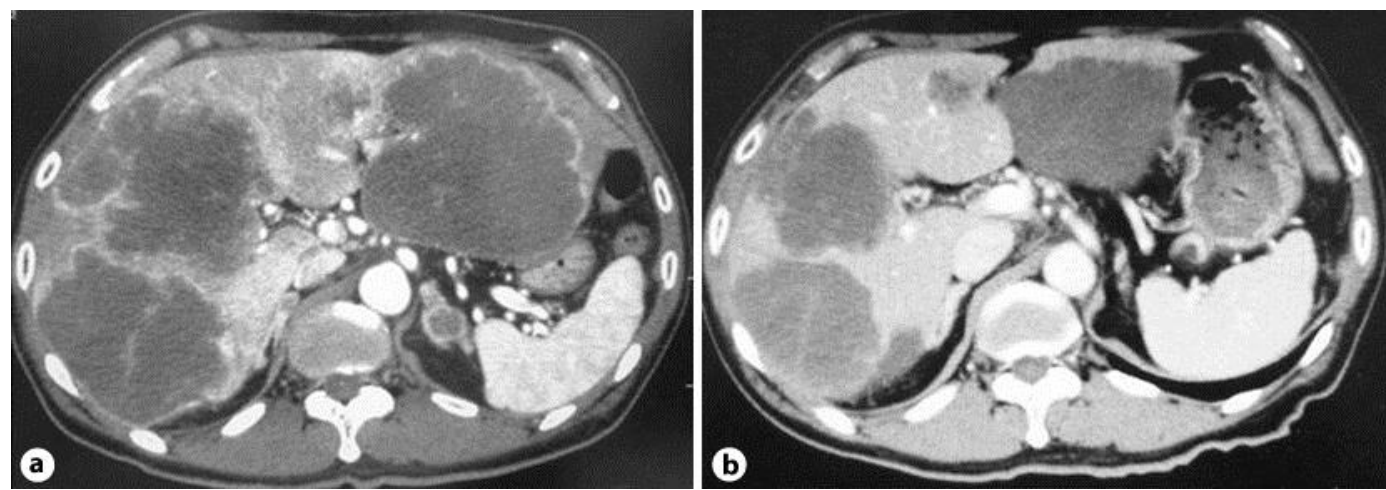

Fig. 2. Abdominal CT. a Imaging before chemotherapy: multiple liver metastases are noted by CT. b Imaging after 4 cycles of chemotherapy: CT revealed a reduction in tumor size. 

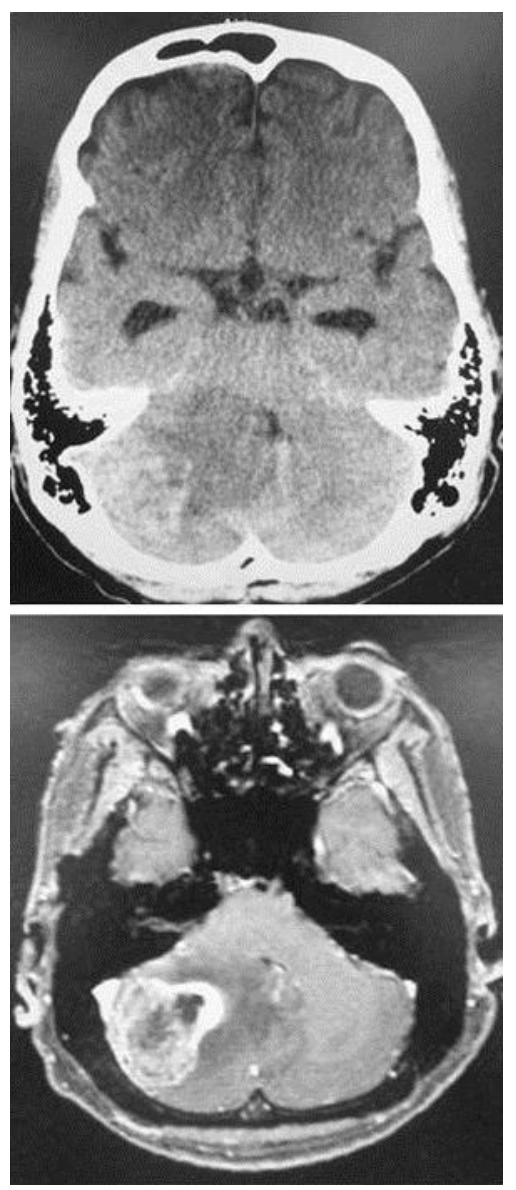

Fig. 3. Head CT and MRI. Head CT and MRI showed a cerebellum metastasis.

\section{References}

1 Schouten LJ, Rutten J, Huveneers HA, Twijnstra A: Incidence of brain metastases in a cohort of patients with carcinoma of the breast, colon, kidney, and lung and melanoma. Cancer 2002;94:2698-2705.

2 Noura S, Ohue M, Shingai T, Fujiwara A, Imada S, Sueda T, Yamada T, Fujiwara Y, Ohigashi H, Yano M, Ishikawa 0: Brain metastasis from colorectal cancer: prognostic factors and survival. J Surg Oncol 2012 (in press).

-3 Joffe J, Gordon PH: Palliative resection for colorectal carcinoma. Dis Colon Rectum 1981;24:355-360.

4 Longo WE, Ballantyne GH, Bilchik AJ, Modlin IM: Advanced rectal cancer. What is the best palliation? Dis Colon Rectum 1988;31:842-847.

5 Rosen SA, Buell JF, Oshida A, Kazsuba S, Hurst R, Michelassi F, Millis JM, Posner MC: Initial presentation with stage IV colorectal cancer: how aggressive should we be? Arch Surg 2000;135:530-534.

-6 Temple LK, Hsieh L, Wong WD, Saltz L, Schrag D: Use of surgery among elderly patients with stage IV colorectal cancer. J Clin Oncol 2004;22:3475-3484.

-7 Kaufman MS, Radhakrishnan N, Roy R, Gecelter G, Tsang J, Thomas A, Nissel-Horowitz S, Mehrotra B: Influence of palliative surgical resection on overall survival in patients with advanced colorectal cancer: a retrospective single institutional study. Colorectal Dis 2008;10:498-502.

-8 Eisenberger A, Whelan RL, Neugut AI: Survival and symptomatic benefit from palliative primary tumor resection in patients with metastatic colorectal cancer: a review. Int J Colorectal Dis 2008;23:559-568. 
9 Scheer MG, Sloots CE, van der Wilt GJ, Ruers TJ: Management of patients with asymptomatic colorectal cancer and synchronous irresectable metastases. Ann Oncol 2008;19:1829-1835.

10 Benoist S, Pautrat K, Mitry E, Rouguer P, Penna C, Nordlinger B: Treatment strategy for patients with colorectal cancer and synchronous irresectable liver metastases. Br J Surg 2005;92:1155-1160.

11 Yoshida Y, Hoshino S, Aisu N, Shiwaku H, Beppu R, Tanimura S, Yamashita Y: Dexamethasone as a means not only for controlling vascular pain caused by the administration of oxaliplatin via the peripheral vein but also for controlling oxaliplatin-induced hypersensitivity reactions. Br J Med Med Res 2012;2:132141

12 Kabbinavar FF, Hambleton J, Mass RD, Hurwitz HI, Bergsland E, Sarkar S: Combined analysis of efficacy: the addition of bevacizumab to fluorouracil/leucovorin improves survival for patients with metastatic colorectal cancer. J Clin Oncol 2005;23:3706-3712.

-13 Cunningham D, Humblet Y, Siena S, Khayat D, Bleiberg H, Santoro A, Bets D, Mueser M, Harstrick A, Verslype C, Chau I, Van Cutsem E: Cetuximab monotherapy and cetuximab plus irinotecan in irinotecanrefractory metastatic colorectal cancer. N Engl J Med 2004;351:337-345.

14 Van Cutsem E, Peeters M, Siena S, Humblet Y, Hendlisz A, Neyns B, Canon JL, Van Laethem JL, Maurel J, Richardson G, Wolf M, Amado RG: Open-label phase III trial of panitumumab plus best supportive care compared with best supportive care alone in patients with chemotherapy-refractory metastatic colorectal cancer. J Clin Oncol 2007;25:1658-1664.

15 Wronski M, Arbit E: Resection of brain metastases from colorectal carcinoma in 73 patients. Cancer 1999;85:1677-1685.

16 Makino T, Mishima H, Ikenaga M, Tsujinaka T, Takeda M, Mano M: Clinicopathologic features of signetring cell carcinoma of the colon and rectum. Jpn J Gastroenterol Surg 2006;39:16-22.

-17 Tajima T, Mukai M, Hinoki T, Ootani Y, Sato S, Nakasaki H, Makuuchi H: A case of poorly differentiated carcinoma of the ascending colon with rapid postoperative progression suggesting disseminated carcinomatosis of the bone marrow. Jpn J Gastroenterol Surg 2006;39:265-270.

18 Yoshida Y, Hoshino S, Shiwaku H, Beppu R, Tanimura S, Tanaka S, Yamashita Y: Early start of chemotherapy after resection of primary colon cancer with synchronous multiple liver metastases: a case report. Case Rep Oncol 2011;4:250-254. 